
FRI0693 FEARS AND MISCONCEPTIONS OF WOMEN WITH CHRONIC RHEUMATIC DISEASES ON THEIR JOURNEY TO MOTHERHOOD

A. Tincani ${ }^{1}$, P. Taylor ${ }^{2}$, R. Fischer-Betz ${ }^{3}$, C. Ecoffet ${ }^{4}$, E. Chakravarty ${ }^{5} .{ }^{1}$ Università degli Studi di Brescia, Brescia, Italy, ${ }^{2}$ Botnar Research Centre, Oxford, United Kingdom, ${ }^{3}$ Rheumatology \& Hiller Research Unit, University Hospital, Düsseldorf, Germany, ${ }^{4}$ UCB Pharma, Brussels, Belgium, ${ }^{5}$ Oklahoma Medical Research Foundation, Oklahoma City, OK, United States

Background: Recent EULAR 'Points to Consider' provide guidance on management and treatment of Women of Childbearing Age (WoCBA) with chronic rheumatic diseases (CRD: RA, axSpA, PsA). However, it is still unclear if these patients (pts) feel adequately supported to make informed treatment decisions around pregnancy and breastfeeding

Objectives: To gain insight into perspectives of women with CRD regarding disease management and pregnancy, and assess whether current clinical practice provides adequate support.

Methods: WoCBA (aged 18-45 years) from Germany, France, UK, Italy, Spain (EU5), the US and Japan participated in a 20-min online survey (Jul-Oct 2017; InSites Consulting). We report data from pts with moderate-severe CRD who were pregnant or had been pregnant in the past $2-5$ years.

Results: 622/1052 participants had CRD (RA, $n=298$; axSpA, $n=182 ;$ PsA, $n=142)$ and resided in the EU5 $(n=306)$, US $(n=293)$ and Japan $(n=23) .87 \%$ pts reported having moderate CRD; $49 \%$ WoCBA stated that they had actively planned their most recent pregnancy. Fewer than half $(46 \%)$ of WoCBA visited a healthcare professional (HCP) before pregnancy, of whom $53 \%$ consulted a rheumatologist among other HCPs (figure 1A). Although guidelines recommend addressing family planning/pregnancy in women with CRD before conception, $69 \%$ pts who visited an HCP before pregnancy had to initiate these discussions with their HCPs. 54\% WoCBA admitted delaying their decision to become a mother; their main fear was passing on health issues to their child (figure 1B). $32 \%$ pts reported having inadequately controlled disease activity during pregnancy; $51 \%$ experienced disease improvement, $22 \%$ disease worsening. $82 \%$ pts visited an obstetrician/gynaecologist (OB/GYN) across trimesters, 68\% a rheumatologist among other HCPs (figure 1A); $65 \%$ had a treatment plan aligned between different HCPs. Stopping treatment during pregnancy was largely driven by fear of harming the foetus (78\%). Among the 113 pts on anti-TNFs, $22 \%$ decided to stop treatment themselves at the start of/during pregnancy, and $47 \%$ were advised to stop by their HCP. Although $89 \%$ pts reported discussing breastfeeding with an $\mathrm{HCP}$ (OB/GYNs were most influential), $66 \%$ mothers felt they had to decide between treatment and breastfeeding. While information provided by their HCP was generally satisfactory, pts still felt they lacked information on the impact of treatment decisions on pregnancy (38\%) and breastfeeding (24\%).

Conclusions: Despite current treatment recommendations, WoCBA with CRD continue to have many fears and misconceptions about their journey to motherhood, due to lack of guidance and consistent information regarding family planning, pregnancy and breastfeeding. Survey findings suggest that women's decisions to delay pregnancy and interrupt their treatment may be linked to a need for greater awareness of disease management options to optimise pregnancy outcomes. Access to this information, consultation with specialists and OB/GYNs earlier in the pregnancy planning process, and an aligned treatment plan could help prevent unnecessary decisions.

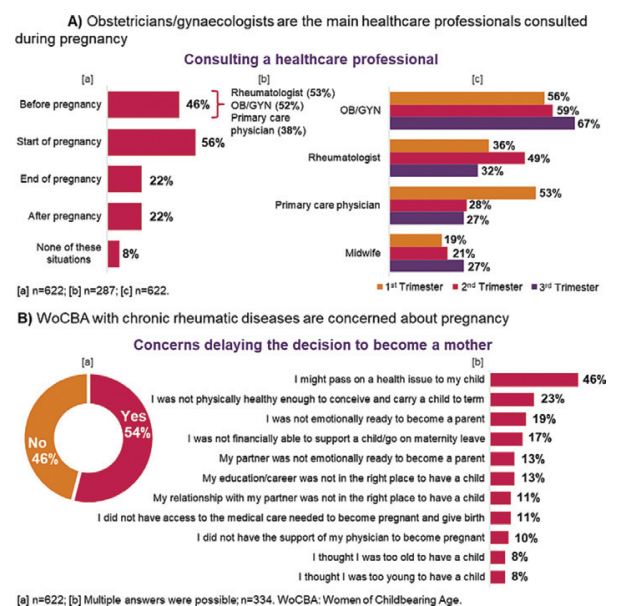

Figure 1A) Obstetricians/gynaecologists are the main healthcare professionals consulted during pregnancy. 1B) WoCBA with chronic rheumatic diseases are concerned about pregnancy [a] $n=622$; [b] Multiple answers were possible; $n=334$. WoCBA: Women of Childbearing Age.
Acknowledgements: This study was funded by UCB Pharma, conducted by InSites Consulting with the help of patient associations, editorial services by Costello Medical. We thank the patients who contributed.

Disclosure of Interest: A. Tincani Grant/research support from: AbbVie, Actelion, Pfizer, UCB Pharma, Consultant for: Celgene and Pfizer, P. Taylor Grant research support from: UCB Pharma, Janssen, Galapagos, Eli Lilly, Abide Therapeutics, Consultant for: Novartis, AbbVie, Eli Lilly, UCB Pharma, Pfizer, Biogen, Janssen, Sanofi, GSK, R. Fischer-Betz Consultant for: AbbVie, BMS, Celgene, Chugai, Novartis, Lilly, UCB Pharma, Pfizer, Janssen, Sanofi, C. Ecoffet Employee of: UCB Pharma, E. Chakravarty Grant/research support from: UCB Pharma

DOI: 10.1136/annrheumdis-2018-eular.2063

\section{FRI0694 \\ TWITTER: A NEW PLATFORM FOR PUBLIC HEALTH CAMPAIGNS: SYSTEMIC LUPUS ERYTHEMATOSUS AND RHEUMATOID ARTHRITIS AS EXAMPLES}

M. Mohameden ${ }^{1}$, B. Alkhairi' ${ }^{2}$, C. Yuvienco ${ }^{1} .{ }^{1}$ Internal Medicine, University of California San Francisco Fresno, fresno, ${ }^{2}$ Internal Medicine, Blake Medical Center, Bradenton, United States

Background: Social media is widely used these days and has the capacity to distribute information widely and rapidly to a large audience. Twitter is a popula social network site with hundreds of millions of users and over 500 million Tweets being sent each day. Patients commonly receive information about their condition from social media sites including Twitter. Patient education plays an important role in the management of systemic lupus erythematosus (SLE) and rheumatoid arthritis (RA). The role of Twitter and its present application in SLE and RA patients' education was analyzed.

Methods: Twitter advanced search function was used to retrieve all tweets (Eng lish language only) containing the words systemic lupus erythematosus, lupus and/or SLE, as well as rheumatoid arthritis over one week (October 23, 2017 to October 29, 2017). Contents of collected tweets were analyzed, and tweets were categorized into 2 groups: general public (editorial/blogging, social/conversational, and advertisement tweets) and professional sources (tweets from physicians, rheumatologists, including hyperlinks to academic publications and websites of professional organizations, and educational tweets by awareness groups).

Results: Over the one-week timeframe, a total of 618 tweets mentioned lupus, systemic lupus erythematosus and/or SLE, and a total of 178 tweets mentioned rheumatoid arthritis. In the respective disease groups, tweets assigned to the professional sources category were $32 \%$ of the total tweets for SLE (200/618) and $51 \%$ for RA (92/178). Tweets sent by physicians constituted $2.6 \%$ (21/796) and by rheumatologists $1 \%(8 / 796)$ of the total tweets. Tweets with links to reliable sources (defined as hyperlinks to academic publications, professional organizations and awareness groups) were $23 \%$ of the total tweets. Finally, educational tweets by patient advocate groups were $10 \%$ of the total tweets.

Conclusions: Twitter has the capability of engaging a wide audience in a topicspecific conversation. Thus, it can be used as cost-effective platform for public health campaigns and to distribute evidence-based knowledge to educate patients. At this time, disease-specific search revealed a smaller percentage of content is employed for public health education and awareness. Links to reliable sources were $23 \%$ of the total tweets, indicating shortage of contribution from healthcare organizations and professionals. Majority of tweets were in the general public category (including tweets by patients) indicating an audience waiting for professional input. Health advocates should use Twitter to construct public health campaigns. Further research should be conducted to examine approaches to target specific Twitter users that engage in SLE and RA conversations.

Disclosure of Interest: None declared DOI: 10.1136/annrheumdis-2018-eular.1996

\section{FRI0695 \\ DMARD MONITORING GUIDELINES AND SHARED PRESCRIBING IN PRIMARY CARE - IS MORE EDUCATION REQUIRED?}

G. Choudhury ${ }^{1}$, M. K. Nisar ${ }^{1} .{ }^{1}$ Rheumatology, Luton \& Dunstable University Hospital, Luton, United Kingdom

Background: DMARDs remain the mainstay of inflammatory arthritis therapy. Though an effective treatment, these drugs have the potential to cause serious harm. Vigilant drug monitoring, reflected in recently updated BSR guidelines, is necessary to prevent potentially life threatening complications. National Patient Safety Agency has also issued several alerts regarding DMARDs and methotrexate overdose remains a 'never event'. Prescribing medications, including DMARDs for rheumatology patients, is one of the chief responsibilities of primary care doctors. 